\title{
Prevalence, clinical characteristics, and predictors of peripheral arterial disease in hemodialysis patients: a cross-sectional study
}

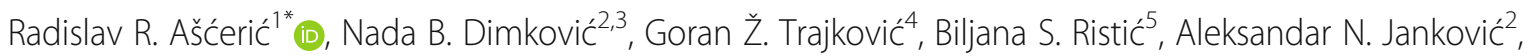
Petar S. Durić ${ }^{2}$ and Nenad S. llijevski,

\begin{abstract}
Background: Peripheral arterial disease (PAD) is common in patients with end-stage renal disease on hemodialysis, but is frequently underdiagnosed. The risk factors for PAD are well known within the general population, but they differ somewhat in hemodialysis patients. This study aimed to determine the prevalence of PAD and its risk factors in patients on hemodialysis.

Methods: This cross-sectional study included 156 hemodialysis patients. Comorbidities and laboratory parameters were analyzed. Following clinical examinations, the ankle-brachial index was measured in all patients. PAD was diagnosed based on the clinical findings, ankle-brachial index $<0.9$, and PAD symptoms.

Results: PAD was present in 55 of $156(35.3 \%$; $95 \%$ Cl, 27.7-42.8\%) patients. The patients with PAD were significantly older (67 \pm 10 years vs. $62 \pm 11$ years, $p=0.014)$, more likely to have diabetes mellitus $(p=0.022)$, and anemia $(p=0.042)$, and had significantly lower serum albumin $(p=0.005)$, total cholesterol $(p=0.024)$, and iron $(p=0.004)$ levels, higher glucose $(p=0.002)$ and $C$-reactive protein $(p<0.001)$ levels, and lower dialysis adequacies $(p=0.040)$ than the patients without PAD. Multivariate analysis showed higher $C$-reactive protein level (odds ratio [OR], 1.03; 95\% confidence interval $[\mathrm{CI}], 1.00-1.06 ; p=0.030$ ), vascular access by Hickman catheter $(\mathrm{OR}, 4.66 ; 95 \% \mathrm{Cl}, 1.03-21.0 ; p=0.045)$, and symptoms of $\mathrm{PAD}(\mathrm{OR}, 5.20 ; 95 \% \mathrm{Cl}, 2.60-10.4 ; p<0.001)$ as independent factors associated with PAD in hemodialysis patients.

Conclusion: The prevalence of PAD was high among patients with end-stage renal disease on hemodialysis. Symptoms of PAD, higher C-reactive protein levels, and Hickman vascular access were independent predictors of PAD in patients on hemodialysis.
\end{abstract}

Keywords: Peripheral arterial disease, Ankle-brachial index, Hemodialysis, C-reactive protein, Hickman vascular access

\section{Introduction}

Peripheral arterial disease (PAD) of the lower extremities is widespread, and it affects over 200 million people worldwide [1]. PAD is also highly prevalent among patients with end-stage renal disease, and it has serious consequences that influence patient outcomes $[2,3]$. The prevalence of PAD has increased over the last

\footnotetext{
* Correspondence: radislavasceric@gmail.com

${ }^{1}$ Department of Vascular Surgery Clinic of Surgery, Clinical Hospital Center

Zvezdara, Dimitrija Tucovića 161, Belgrade 11000, Serbia

Full list of author information is available at the end of the article
}

decade, particularly in low-income countries [4]; further, it increases with age and is $4.5-14.5 \%$ more prevalent among individuals aged $\geq 65$ years [5]. Among patients on hemodialysis (HD), the prevalence of PAD is much higher, ranging from 17 to $48 \%[6,7]$.

The findings from the Dialysis Outcomes and Practice Patterns Study (DOPPS) described a PAD prevalence of $25.3 \%$ among patients on HD that was accompanied by significant geographic variations. In European countries, PAD prevalence was $17.5-37.8 \%$, while in Japan, the prevalence was significantly lower at $11.5 \%$ [8]. Similarly,

(C) The Author(s). 2019 Open Access This article is distributed under the terms of the Creative Commons Attribution 4.0 International License (http://creativecommons.org/licenses/by/4.0/), which permits unrestricted use, distribution, and 
the findings from the Hemodialysis Study (HEMO) showed that PAD was seen in $23 \%$ of patients on HD [9]. The findings from the National Health and Nutrition Examination Survey (NHANES) 1999-2000 showed that PAD was present in $24 \%$ of patients with renal insufficiency and in $3.7 \%$ of participants with normal renal function; these findings were independent of diabetes, age, and hypertension [10]. Japanese investigators have shown that PAD prevalence in patients on HD ranges from 23.8 to $47 \%$, depending on the study population $[2,11]$. Although these studies were valuable, they had different methods for PAD diagnosis.

The early diagnosis and management of PAD can improve the prognosis for patients on HD [11]. Therefore, many patients on HD with PAD could avoid or at least delay adverse events, such as amputations, cardiovascular events, and death if PAD was timely diagnosed and adequately treated [12]. The traditional risk factors for PAD, namely, age, diabetes mellitus (DM), smoking, hyperlipidemia, hypertension, and male sex, are well recognized within the general population [7], but these risk factors are supplemented by other risk factors in patients on HD $[13,14]$. To our knowledge, no studies have previously investigated the effects of vascular access on PAD prevalence. Thus, this study aimed to examine the prevalence of PAD, the risk factors associated with PAD, and the clinical characteristics of PAD in patients on HD.

\section{Methods}

\section{Study population}

The study subjects comprised 197 outpatients who were on maintenance $\mathrm{HD}$ at the Clinical Department for Nephrology and Metabolic Disorders with Dialysis at Zvezdara University Medical Center. Of these subjects, 41 patients were excluded from the study because 36 did not provide informed consent and five died during the study and their data were incomplete. Hence, 156 patients participated in this cross-sectional study between January 2016 and January 2017. Zvezdara University Medical Center's ethics committee approved this study, and all participants provided written informed consent before study initiation.

\section{Risk factors and comorbid diseases}

The patients' medical records were reviewed, and data describing their demographic and clinical characteristics were collected, including age, sex, height, weight, HD duration and type, and causes of renal failure. The following risk factors and comorbidities associated with PAD were analyzed: smoking habits, hypertension, hyperlipidemia, DM, anemia, coronary artery disease, and cerebrovascular diseases, including stroke. The body mass index was calculated as the weight in kilograms divided by height in meters squared.

\section{Laboratory parameters}

Blood samples were taken quarterly at the beginning of the second HD session of the week. The analyzed laboratory parameters included the serum creatinine, urea, potassium, calcium, albumin, phosphorus, total cholesterol, triglyceride, glucose, C-reactive protein (CRP), iron, transferrin saturation, ferritin, intact parathyroid hormone, dialysis adequacy $(\mathrm{Kt} / \mathrm{V})$, hemoglobin levels and blood cell counts. Mean values of four measurements were used in the analyses.

\section{Patient assessments and clinical examinations}

Blood pressure measurements were obtained before and after dialysis and during three consecutive dialysis sessions, and the mean values were used to calculate the pulse pressure (systolic pressure - diastolic pressure) and the mean arterial pressure (diastolic pressure $+1 / 3$ pulse pressure). The presence of left ventricular hypertrophy was determined indirectly using electrocardiography.

The patients' histories of previous peripheral artery interventions, including bypass surgery, percutaneous interventions, or amputations for PAD, were documented. The patients were asked about PAD symptoms, including claudication, coldness, numbness, and resting pain, and they were categorized using the Fontaine classification of PAD symptoms [15].

All patients underwent standard physical examinations that included pulse palpation on the femoral artery, popliteal artery, dorsalis pedis artery, and posterior tibial artery on both legs, and auscultation for femoral bruits. The pulses were coded as absent (0), weak (1), or present (2). To ensure that the findings were comparable, all patients were examined by the same experienced physician.

\section{Ankle-brachial index ( $\mathrm{ABI}$ ) measurements}

We measured ABI using a portable handheld bidirectional Doppler device (Life Dop 250 ABI; Wallach Surgical Devices, Trumbull, CT, USA). All ABIs were measured at room temperature and before dialysis while the patients were unclothed, in a supine position, and after they had rested for 5-10 min. The systolic blood pressure was measured in the brachial artery of the arm without vascular access and in the dorsalis pedis artery and posterior tibial artery of the right and left lower limbs after using a Doppler probe of appropriate cuff size. The same well-trained nurse performed all ABI measurements. The ABI was calculated by dividing the ankle systolic pressure by arm systolic pressure. The lower ABI value of both legs was used as the leg index. 
An ABI was classified as low $(<0.9)$ if any of the $A B I$ values were $<0.9$, normal $(0.9-1.3)$ if all four values were between 0.9 and 1.3, and high $(>1.3)$ if at least one ABI was $>1.3$ with other values being normal [16].

Patients were diagnosed with PAD if they fulfilled the criteria relating to clinical examinations, Fontaine classification, and ABIs. The patients were considered to have PAD if they had an ABI $<0.9$ or if they had claudication and absence of pulses, despite having normal ABIs. We performed duplex ultrasonography on patients who had normal and high ABIs, and confirmed PAD if there was $\geq 50 \%$ narrowing of the large lower extremity blood vessels that had caused either sub-occlusion or occlusion of $\geq 1$ crural arteries.

\section{Statistical analyses}

The R software environment for statistical computing [17] was used to conduct the statistical analyses. The significance level was set at 0.05 . Continuous variables are presented as the means and standard deviations, and categorical variables are presented as numbers and percentages; these descriptive statistics were used to characterize the study sample. The differences between groups in relation to the continuous variables were analyzed using Student's t-test and the Mann-Whitney U test for the independent samples. Pearson's chi-squared test or Fisher's exact test were used to analyze the differences between groups in relation to the categorical variables. A multivariate logistic regression model was constructed to determine the predictors of PAD, which included the statistically significant variables $(P<0.05)$ from the univariate analyses.

\section{Results}

There were no statistically significant differences between patients who did and did not participate in the study in terms of age, sex, and HD vintage. Of the 156 patients identified, PAD was present in 55 (35.3\%; 95\% CI, 27.7-42.8\%), of which $40 \%$ were women (Table 1 ). Hypertension was the most common condition that caused the end-stage renal disease (53.2\%), and DM was the second most common cause of the end-stage renal disease (12.8\%) in our patients. Compared with the nonPAD group, the PAD patients were older, more likely to be male, and had higher incidence rates of anemia and DM. The participants' mean HD vintage was $71.7 \pm 69.5$ (median: 55; range: 0-354) months. The groups did not differ in relation to the HD type and daily HD duration. Type of vascular access significantly differed between the PAD and non- PAD groups $(p=0.004)$. The prevalence of PAD was the highest in patients with Hickman vascular catheter (75\%), followed by patients with arteriovenous graft (AVG; 53.8\%), and was the lowest in patients with arteriovenous fistula (AVF; 30\%). The only statistically significant difference in PAD prevalence between these groups was found when patients with Hickman vascular catheter and AVF were compared $(p=0.003)$. Although the prevalence of PAD was greater in patients with AVG than in those with AVF, there was no significant difference $(p=0.117)$.

The PAD group had a lower median $\mathrm{Kt} / \mathrm{V}$ value than the non-PAD group (1.31 [range: $0.82-2.14$ ] vs. 1.37 [range: $0.51-3.34$ ]; $p=0.04$ ). Two patients had undergone major amputations as a consequence of PAD; specifically, one had above-the-knee amputation and one had below-the-knee amputation. Four patients had undergone minor amputations, comprising two who had amputations of one toe and two who had amputations of a little toe. One patient had undergone an abdominal aortic aneurysm resection and another patient had undergone a femoro-femoral cross-over bypass. The patients in the PAD and nonPAD groups did not differ in relation to the systolic blood pressure, diastolic blood pressure, pulse pressure, and mean arterial pressure values determined before and after HD.

The prevalence of PAD was associated with the age of patients on HD, and it was most prevalent among patients who were $\geq 80$ years old (Fig. 1).

Table 2 presents the participants' symptoms and clinical findings. Of the patients on HD without PAD, 19.8\% had various symptoms in their legs, including coldness and numbness, and $2 \%$ had claudications. The PAD and non-PAD groups differed significantly in relation to the clinical findings, including pulse palpation, femoral bruit auscultation, and ABI values (all $p<0.001$ ).

The patients on HD with PAD had significantly lower median ABIs than the patients on HD without PAD (0.90 vs. $1.13 ; p<0.001$ ) (Fig. 2 ).

Table 3 summarizes the laboratory blood test results. The albumin, total cholesterol, and iron levels were lower and the glucose and CRP levels were higher in the PAD group than in the non-PAD group.

Table 4 presents the results of logistic regression analyses using PAD as the dependent variable. The univariate analysis indicated that age, DM, symptoms based on the Fontaine classification, vascular access provided by a Hickman catheter, lower albumin and total cholesterol levels, and a higher CRP level were associated with PAD (all $p<0.05)$. The multivariate logistic regression analysis showed that the independent factors associated with PAD in patients on HD were a higher CRP level (odds ratio [OR], 1.03; 95\% confidence interval $[\mathrm{CI}], 1.00-1.06 ; p=0.030$ ), vascular access provided by a Hickman catheter (OR, 4.66; 95\% CI, 1.03-21.0; $p=0.045)$, and presence of PAD symptoms based on the Fontaine classification (OR, 5.20; 95\% CI, 2.60-10.4; $p<0.001)$. 
Table 1 Demographic, baseline and dialysis characteristics of the study population

\begin{tabular}{|c|c|c|c|c|}
\hline \multirow[t]{2}{*}{ Characteristics } & \multirow{2}{*}{$\begin{array}{l}\text { Total } \\
156(100)\end{array}$} & \multirow{2}{*}{$\begin{array}{l}\text { PAD } \\
55(35.3)\end{array}$} & \multirow{2}{*}{$\begin{array}{l}\text { No PAD } \\
101(64.7)\end{array}$} & \multirow[t]{2}{*}{$p$} \\
\hline & & & & \\
\hline Age years mean $\pm S D$ & $64 \pm 11$ & $67 \pm 10$ & $62 \pm 11$ & 0.014 \\
\hline Gender, n (\%) & & & & 0.905 \\
\hline Male, n (\%) & $96(61.5)$ & $33(60.0)$ & $63(62.4)$ & \\
\hline Primary kidney disease, n (\%) & & & & 0.118 \\
\hline Hypertension & $83(53.2)$ & $28(50.9)$ & $55(54.5)$ & \\
\hline Diabetes mellitus & $20(12.8)$ & $12(21.8)$ & $8(7.9)$ & \\
\hline Glomerulonephritis & $18(11.5)$ & $4(7.3)$ & $14(13.9)$ & \\
\hline Cystic disease & $12(7.7)$ & $2(3.6)$ & $10(9.9)$ & \\
\hline Pyelonephritis and Obstructive uropathy & $10(6.4)$ & $4(7.3)$ & $6(5.9)$ & \\
\hline Other & $13(8.3)$ & $5(9.1)$ & $8(7.9)$ & \\
\hline $\mathrm{BMl}$, mean $\pm \mathrm{SD}$ kg/m2 & $24.0 \pm 4.1$ & $24.2 \pm 4.2$ & $23.9 \pm 4.0$ & 0.68 \\
\hline Hypertension, n (\%) & $142(91.0)$ & $53(96.4)$ & $89(88.1)$ & 0.14 \\
\hline Hyperlipoproteinemia, n (\%) & $75(48.1)$ & $22(40.0)$ & $53(52.5)$ & 0.136 \\
\hline Diabetes mellitus, n (\%) & & & & 0.022 \\
\hline No & $117(75.0)$ & $34(61.8)$ & $83(82.2)$ & \\
\hline Type II oral therapy & $16(10.3)$ & $8(14.5)$ & $8(7.9)$ & \\
\hline Type II Insulin & $20(12.8)$ & $12(21.8)$ & $8(7.9)$ & \\
\hline Type I Insulin & $3(1.9)$ & $1(1.8)$ & $2(2.0)$ & \\
\hline CAD, n (\%) & $54(34.6)$ & $20(36.4)$ & $34(33.7)$ & 0.801 \\
\hline Angina pectoris, n (\%) & $45(28.8)$ & $15(27.3)$ & $30(29.7)$ & 0.750 \\
\hline Myocardial infarction, n (\%) & $14(9.0)$ & $8(14.8)$ & $6(5.9)$ & 0.081 \\
\hline CABG, $\mathrm{PCl}, \mathrm{n}(\%)$ & $15(9.6)$ & $8(14.8)$ & $7(6.9)$ & 0.153 \\
\hline Stroke n (\%) & $6(3.8)$ & $4(7.3)$ & $2(2.0)$ & 0.186 \\
\hline Anemia, n (\%) & $144(92.3)$ & $54(98.2)$ & $90(89.1)$ & 0.042 \\
\hline Smoking, n (\%) & & & & 0.677 \\
\hline No & $82(52.6)$ & $27(49.1)$ & $55(54.5)$ & \\
\hline Yes & $39(25.0)$ & $16(29.1)$ & $23(22.8)$ & \\
\hline Past & $35(22.4)$ & $12(21.8)$ & $23(22.8)$ & \\
\hline LVH, n (\%) & $17(10.9)$ & $5(9.8)$ & $12(12.9)$ & 0.582 \\
\hline Access for HD, n (\%) & & & & 0.004 \\
\hline AVF & $130(83.3)$ & 39 (70.9) & $91(90.1)$ & \\
\hline CVC & $1(0.6)$ & $0(0.0)$ & $1(1.0)$ & \\
\hline Hickman vascular catheter & $12(7.7)$ & $9(16.4)$ & $3(3.0)$ & \\
\hline AVG & $13(8.3)$ & $7(12.7)$ & $6(5.9)$ & \\
\hline HD vintage (months), median (range) & $55(0,354)$ & $55(0,264)$ & $55(0,354)$ & 0.867 \\
\hline Type of HD, n (\%) & & & & 0.086 \\
\hline HDF & 29 (18.6) & $6(10.9)$ & $23(22.8)$ & \\
\hline High flux HD & $127(81.4)$ & $49(89.1)$ & $78(77.2)$ & \\
\hline Kt/N median (range) & $1.34(0.51,3.35)$ & $1.31(0.82,2.14)$ & $1.37(0.51,3.34)$ & 0.040 \\
\hline
\end{tabular}

AVF Arteriovenous fistula, AVG Arteriovenous graft, BMI Body mass index, CAD Coronary artery disease, CABG coronary artery bypass graft, CVC Central venous catheter, HD Hemodialysis, HDF Hemodiafiltration, $L V H$ Left ventricular hypertrophy, PAD Peripheral arterial disease, $P C l$ Percutaneous coronary intervention 


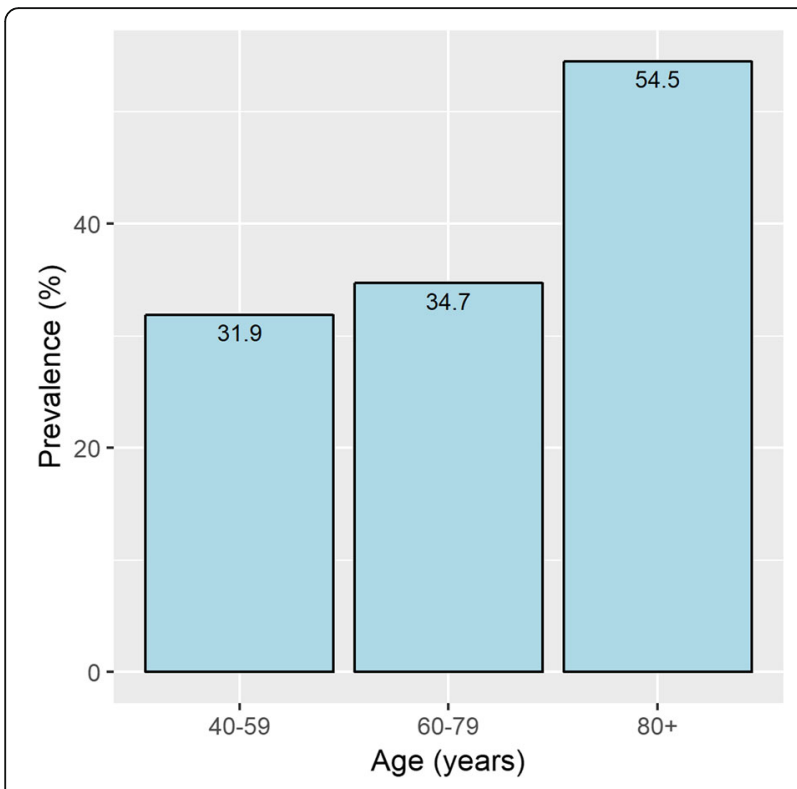

Fig. 1 Prevalence of peripheral arterial disease in the hemodialysis patients according to age. $X$ title: Age (years). Y title: Prevalence (\%)

\section{Discussion}

Approximately $35 \%$ of patients on HD in this study had PAD, which concurs with the rates published to date [6, 7]. In this study, PAD was diagnosed based on an $\mathrm{ABI}$ of $<0.9$, clinical findings, and categorization of symptoms based on the Fontaine classification; 41 patients with PAD had ABI values $<0.9$, seven patients had $\mathrm{ABI}$ values $>1.3$, and seven had ABI values between 0.9 and 1.3, or more specifically, between 0.91 and 1.0. We confirmed PAD in seven patients with normal and in seven patients with high ABI values using color Doppler ultrasonography. There were significant differences between the PAD and non-PAD groups with respect to the clinical findings, including those from pulse palpation and femoral bruit auscultation, and ABIs. The clinical findings were noticeably worse in the PAD group than in the non-PAD group. Often, these patients have DM; chronic kidney disease alone leads to arterial incompatibility as a consequence of pronounced calcification, and arterial stiffness generates false-positive ABIs $[2,6,16]$. An $\mathrm{ABI}<0.9$ alone should not be used to assess the severity of PAD in patients on HD [2]. An ABI of $<0.9$, in conjunction with the clinical findings, namely, absence of pulses and presence of femoral bruits, and PAD symptoms, including claudication, would provide a more robust foundation for a diagnosis of PAD.

Measuring the ABI is simple, reliable, inexpensive, and non-invasive [18]. According to the most recent American Heart Association guidelines, ABIs of 0.91.09 are lower normal values [18]; however, some Japanese authors have recommended ABIs of 1.051.1 as the limit for a diagnosis of PAD in patients on $\operatorname{HD}[2,11]$.
Table 2 Fontaine symptoms and the study participants' clinical findings

\begin{tabular}{|c|c|c|c|c|}
\hline & Total & PAD & No PAD & $P$ \\
\hline \multicolumn{5}{|c|}{ PAD Symptoms Fontaine, n (\%) } \\
\hline I & $96(61.5)$ & $15(27.3)$ & $81(80.2)$ & \multirow[t]{5}{*}{$<0.001$} \\
\hline$\| \mathrm{a}$ & $37(23.7)$ & $19(34.5)$ & $18(17.8)$ & \\
\hline $11 \mathrm{~b}$ & $21(13.5)$ & $19(34.5)$ & $2(2.0)$ & \\
\hline III & $1(0.6)$ & $1(1.8)$ & $0(0.0)$ & \\
\hline IV & $1(0.6)$ & $1(1.8)$ & $0(0.0)$ & \\
\hline \multicolumn{5}{|c|}{ Femoral bruit, n (\%) } \\
\hline Yes & $45(28.9)$ & $28(50.9)$ & $17(16.8)$ & \multirow[t]{2}{*}{$<0.001$} \\
\hline No & $106(67.9)$ & $23(41.8)$ & $83(82.2)$ & \\
\hline \multicolumn{5}{|l|}{ Pulse, n (\%) } \\
\hline \multicolumn{5}{|l|}{ Femoral } \\
\hline No & $1(0.6)$ & $1(1.8)$ & $0(0)$ & \multirow[t]{3}{*}{0.042} \\
\hline Weak & $2(1.3)$ & $2(3.6)$ & $0(0)$ & \\
\hline Yes & $153(98.1)$ & $52(94.5)$ & $101(100)$ & \\
\hline \multicolumn{5}{|l|}{ Popliteal } \\
\hline No & $22(14.1)$ & $22(40.0)$ & $0(0)$ & \multirow[t]{3}{*}{$<0.001$} \\
\hline Weak & $8(5.1)$ & $7(12.7)$ & $1(1)$ & \\
\hline Yes & $126(80.8)$ & $26(47.3)$ & $100(99)$ & \\
\hline \multicolumn{5}{|l|}{ DPA } \\
\hline No & $46(29.5)$ & $37(67.3)$ & $9(8.9)$ & \multirow[t]{3}{*}{$<0.001$} \\
\hline Weak & 31 (19.9) & $9(16.4)$ & $22(21.8)$ & \\
\hline Yes & 79 (50.6) & $9(16.4)$ & 70 (69.3) & \\
\hline \multicolumn{5}{|l|}{ PTA } \\
\hline No & $50(32.1)$ & $41(74.5)$ & 9 (8.9) & \multirow[t]{3}{*}{$<0.001$} \\
\hline Weak & $39(25.0)$ & $11(20.0)$ & $28(27.7)$ & \\
\hline Yes & $67(42.9)$ & $3(5.5)$ & $64(63.4)$ & \\
\hline \multicolumn{5}{|c|}{ ABI indexes $n(\%)$} \\
\hline $\mathrm{ABI}<0.9$ & $41(26.3)$ & $41(74.5)$ & $0(0.0)$ & \multirow[t]{3}{*}{$<0.001$} \\
\hline$A B \mid>1.3$ & $36(23.1)$ & $7(12.7)$ & $29(28.7)$ & \\
\hline$A B \mid 0.91-1.3$ & 79 (50.6) & $7(12.7)$ & 72 71.3) & \\
\hline
\end{tabular}

$A B I$ Ankle-brachial index, DPA Dorsalis pedis artery, PAD Peripheral arterial disease, TPA Tibialis posterior artery

Intermittent claudication is the classic and most striking symptom associated with PAD, and it is relatively rare in the general population and in patients on HD $[5,19]$. Therefore, intermittent claudication alone is not a reliable criterion for a diagnosis of PAD [6]. The findings from preliminary studies have shown that $20-50 \%$ of patients with PAD who had ABIs of $<0.9$ did not report claudication; hence, these patients belong to the asymptomatic group of patients with PAD [5, 20]. In the UK, Webb et al. [21] studied 325 patients receiving HD and reported that $19 \%$ of these patients had claudication. In this study, 37.2\% of the patients had claudication. In the DOPPS and 


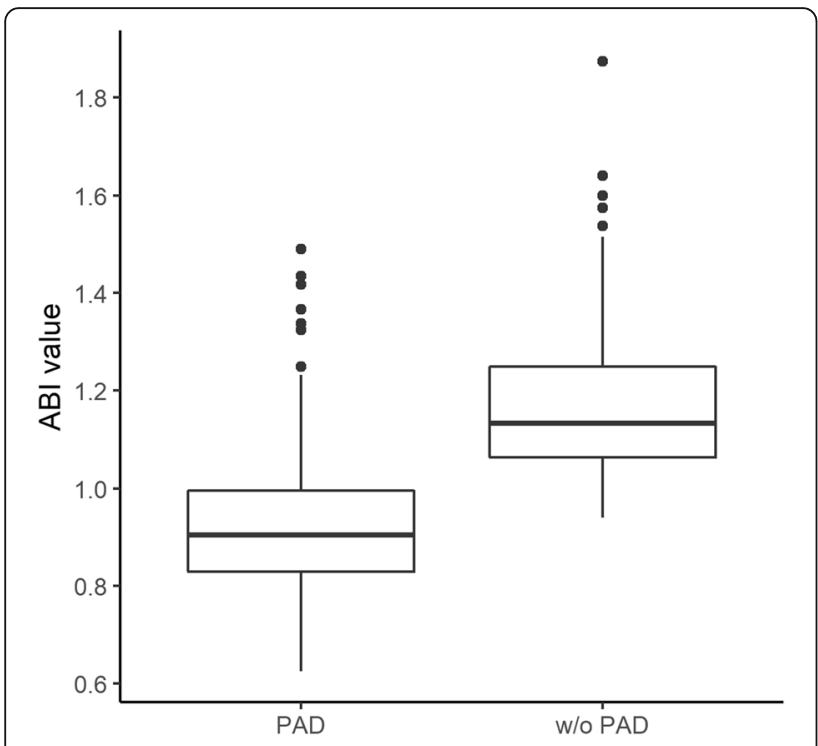

Fig. 2 The median ankle-brachial index values in the hemodialysis patients with and without peripheral arterial disease. $X$ title: PAD, w/ o PAD. Y title: $A B$ I value

HEMO studies of patients on HD, a diagnosis of PAD was based on a previous PAD diagnosis, the presence of intermittent claudication or critical ischemia of the extremities, previous surgical revascularization or amputation as a consequence of PAD, and prior diagnosis of an abdominal aortic aneurysm. Based on these criteria, the rates of PAD in the DOPPS and HEMO studies were 25.3 and $23 \%$, respectively $[8,12]$.
Likewise, the prevalence of PAD in the NHANES was $24 \%$ in the group of patients with chronic kidney disease, and the same criteria were used to diagnose PAD [10].

In studies that used $\mathrm{ABI}<0.9$ as a criterion for PAD diagnosis in patients on HD, PAD prevalence was slightly higher and it reached 37\% [6]. Because ABIs $<0.9$ are relevant for a diagnosis of PAD, they are an important prognostic factor associated with cardiovascular disease and mortality in patients on $\operatorname{HD}[11,16,19]$.

In our study, PAD was associated with older age, DM, and anemia, and a high percentage of patients with PAD had hypertension; however, the rate of left ventricular hypertrophy was not significantly higher in this group of patients, perhaps because it was determined by electrocardiography. Significant differences did not exist with respect to the systolic and diastolic blood pressure values determined before and after HD nor in relation to the pulse pressure and mean arterial pressure values in this study, but other investigators have described significantly higher diastolic and pulse pressure values in patients with PAD on HD [12, 13]. Viazzi et al. [3] suggested that the predictive power associated with ambulatory interdialytic blood pressure monitoring was much greater than the predictive powers of blood pressure measurements attained in the clinic or those determined immediately before and after dialysis. Our patients on HD were closely monitored and their blood pressure was well controlled, regardless of the presence of PAD.

Table 3 Biochemical characteristics of the study population

\begin{tabular}{|c|c|c|c|c|}
\hline & Total & PAD & No PAD & $P$ \\
\hline Urea, mmol/l & $23.1 \pm 4.7$ & $23.3 \pm 5.1$ & $22.9 \pm 4.4$ & 0.663 \\
\hline Creatinine, $\mu \mathrm{mol} / \mathrm{l}$ & $840 \pm 164$ & $837 \pm 182$ & $841 \pm 155$ & 0.878 \\
\hline Albumin, g/L & $37 \pm 2$ & $36 \pm 2$ & $38 \pm 2$ & 0.005 \\
\hline Glucose, mmol/l & $6.4 \pm 1.9$ & $7.0 \pm 2.1$ & $6.0 \pm 1.7$ & 0.002 \\
\hline Total cholesterol, mmol/l & $4.2 \pm 1.0$ & $4.0 \pm 0.8$ & $4.4 \pm 1.1$ & 0.024 \\
\hline Triglyceride, mmol// & $1.83 \pm 0.93$ & $1.82 \pm 1.07$ & $1.83 \pm 0.85$ & 0.934 \\
\hline Erythrocytes, $\times 10^{12} / \mathrm{L}$ & $3.42 \pm 0.30$ & $3.41 \pm 0.29$ & $3.43 \pm 0.30$ & 0.663 \\
\hline Hemoglobin, g/dL & $10.4 \pm 0.9$ & $10.3 \pm 0.8$ & $10.4 \pm 1.0$ & 0.373 \\
\hline Iron, $\mu$ mol//I mean $\pm S D$ & $10.9 \pm 3.3$ & $9.9 \pm 3.0$ & $11.5 \pm 3.3$ & 0.004 \\
\hline T-Sat.,\% median (range) & $0.29(0.08-7.49)$ & $0.28(0.08-0.54)$ & $0.29(0.15-7.49)$ & 0.064 \\
\hline Ferritin, ng/mL median (range) & $279(28-1126)$ & $285(44-906)$ & $274(28-1126)$ & 0.201 \\
\hline White blood cell, $\times 10^{9} / \mathrm{L}$ & $7.05 \pm 2.25$ & $7.47 \pm 2.19$ & $6.82 \pm 2.26$ & 0.086 \\
\hline CRP, mg/L median (range) & $7.3(0.4-104.3)$ & $9.7(0.9-104.3)$ & $6.6(0.4-64.9)$ & $<0.001$ \\
\hline $\mathrm{K}$, mean $\pm \mathrm{SD}$ & $5.22 \pm 0.98$ & $5.20 \pm 1.40$ & $5.24 \pm 0.65$ & 0.775 \\
\hline Calcium, mmol/l & $2.27 \pm 0.17$ & $2.26 \pm 0.15$ & $2.27 \pm 0.17$ & 0.732 \\
\hline Phosphorus, mmol// & $1.50 \pm 0.42$ & $1.51 \pm 0.43$ & $1.48 \pm 0.41$ & 0.74 \\
\hline Parathormone, pg/ml, median (range) & $131(6.1-4184)$ & $123(14.6-1564.6)$ & $140(6.1-4184.0)$ & 0.292 \\
\hline
\end{tabular}

CRP C-reactive protein, PAD Peripheral arterial disease, $T$-sat Transferrin saturation 
Table 4 Logistic regression analyses using peripheral arterial disease as the dependent variable

\begin{tabular}{|c|c|c|c|c|}
\hline \multirow[b]{2}{*}{ Independent variable } & \multicolumn{2}{|l|}{ Univariate model } & \multicolumn{2}{|c|}{ Multivariate model } \\
\hline & OR $(95 \% \mathrm{Cl})$ & $P$ & OR $(95 \% \mathrm{Cl})$ & $P$ \\
\hline Age & $1.04(1.01-1.07)$ & 0.004 & $1.01(0.97-1.06)$ & 0.536 \\
\hline Diabetes mellitus & $2.85(1.35-6.00)$ & $<0.001$ & $1.29(0.48-3.51)$ & 0.817 \\
\hline Anemia & $0.15(0.02-1.21)$ & 0.074 & $0.31(0.03-3.00)$ & 0.313 \\
\hline PAD symptoms by Fontaine & $6.55(3.55-12.1)$ & $<0.001$ & $5.20(2.60-10.4)$ & $<0.001$ \\
\hline Access Hickman & $4.74(1.39-16.2)$ & 0.013 & $4.66(1.03-21.0)$ & 0.045 \\
\hline $\mathrm{Kt} / \mathrm{V}$ & $0.36(0.13-1.06)$ & 0.064 & $1.04(0.27-4.07)$ & 0.953 \\
\hline Albumin & $0.84(0.74-0.96)$ & 0.008 & $1.04(0.88-1.25)$ & 0.627 \\
\hline Total cholesterol & $0.64(0.43-0.94)$ & 0.023 & $0.83(0.50-1.37)$ & 0.462 \\
\hline CRP & $1.04(1.02-1.07)$ & 0.002 & $1.03(1.00-1.06)$ & 0.030 \\
\hline
\end{tabular}

$C R P$ C-reactive protein, $P A D$ Peripheral arterial disease

Several authors have described higher frequencies of cardiovascular disease and stroke among patients with PAD [5, 22]. Although the patients in this study had high rates of cardiovascular disease and stroke, the groups did not differ with regard to their frequencies. Anemia was present among patients with PAD, a finding that concurs with the findings from a previously published study that showed that patients with PAD with anemia had a more severe form of the disease and had higher risks of mortality and limb amputation than patients with PAD without anemia [23].

Whereas the traditional risk factors associated with PAD, namely, age, DM, smoking status, hypertension, male sex, and hyperlipidemia [8], are valid within the general population, the risk factors for PAD slightly differ among patients on HD $[6,13]$. In addition to the traditional risk factors, renal-specific factors are associated with PAD in patients on $\operatorname{HD}[6,12,13]$. A smaller number of studies have investigated correlations between PAD and its risk factors in patients on HD [24]. The findings from the HEMO study that included 936 patients on HD, showed that DM, age, and smoking status are associated with PAD, while the other conventional risk factors, namely, male sex, hypertension, and hypercholesterolemia, are not associated with PAD [9].

The findings from the United States Renal Data System study and the Dialysis Morbidity and Mortality Study, which examined the associations among the variables related to dialysis and the conventional cardiovascular risk factors for PAD in patients on dialysis, showed that a diagnosis of PAD was associated with age, male sex, being Caucasian, DM, smoking status, higher diastolic blood pressure, and left ventricular hypertrophy. In an adjusted analysis, PAD was also associated with low serum parathyroid hormone levels, a longer dialysis vintage, a lower $\mathrm{Kt} / \mathrm{V}$, and a low serum albumin level [25]. Compared with the non-PAD group, the HD vintage was shorter in the PAD group in this study, but the difference was not significant, which concurs with the findings from a study carried out in Japan [12]; however, other investigators consider that PAD is related to the dialysis vintage [14]. Lower Kt/V values were observed among patients in the PAD group, which is consistent with the findings from other studies [11, 25]. These findings might help explain why some uremic toxins that are not removed by dialysis, for example, dimethylarginine, accumulates, damages endothelial cells, and causes atherogenesis [19].

Chronic uremia is associated with systemic inflammation that causes hypoalbuminemia and increases the risk of PAD $[13,26]$, and may play an important role in the pathogenesis and progression of atherosclerosis [13]. Chronic inflammation, malnutrition, and hyperphosphatemia are associated with PAD in patients with end-stage renal disease $[6,7]$. The serum albumin and total cholesterol levels indicate not only a patient's nutritional status but also chronic inflammation $[25,26]$. Serum CRP is related not only to vascular inflammatory reactions but also promotes atherogenesis and atherothrombosis [27]. In this study, patients with PAD had significantly higher CRP levels and lower albumin, total cholesterol, and iron levels, which are indirect indicators of malnutrition, inflammation, and atherosclerosis syndrome [28].

Similar to our study, several investigators have shown that hyperlipidemia is not a risk factor for PAD in patients on HD [12, 29], but some authors have described hyperlipidemia as a risk factor for PAD $[5,11]$. In contrast, the findings from one study indicated that hyperlipidemia had a protective effect against PAD [29].

The patients with PAD in our study had significantly higher levels of glycemia, which correlated with the higher incidence of diabetes in these patients. Several authors have described DM and poor glycemic control as important risk factors for PAD [5, 8, 13, 26]. Earlier reports have suggested that inflammation plays a key 
role in atherosclerosis and confirmed that patients with elevated CRP and lower albumin levels have high occurrence rates of PAD [27]. The findings from this study concur with those from previous studies.

A distal radiocephalic AVF is the vascular access of choice for HD at our center because it is associated with the longest survival times and the lowest number of complications. Patients with inadequate blood vessels undergo AVG implantations. Tunneled Hickman vascular catheters are used for permanent vascular access in patients who have poor prognoses and shorter life expectancies and whose blood vessels are inappropriate for an AVF or AVG implantations [30]. Our data showed that compared with the patients without Hickman vascular catheters $(25 \%)$, a significantly higher number of patients on HD with Hickman vascular catheters had PAD (75\%). We also observed that patients with PAD and Hickman vascular catheter had significantly lower hemoglobin $(p=0.001)$, and albumin $(p=0.022)$ levels than patients with AVF. Compared to the patients with AVF, patients with implanted Hickman vascular catheter and AVG had significantly higher CRP values $(p=0.05)$. The presence of a permanent vascular catheter was a predictor of $\mathrm{PAD}$, and the use of the catheter may be associated with silent or overt inflammation. As is already known, there is a strong correlation between the atherosclerosis of blood vessels of the arm and PAD. Moreover, an increased incidence of vascular access failure was noted in patients with AVG compared to those with AVF [16, 31].

The management and treatment of patients with PAD requires a multidisciplinary approach [32]. The National Kidney Foundation's Kidney Disease Outcomes Quality Initiative guidelines [33] recommend that at the beginning of a dialysis session, all patients should be screened for PAD, be clinically examined, and have their ABIs measured. These patients often have insufficient daily activity and diabetic neuropathy, and they are usually asymptomatic; due to this, they are underdiagnosed and undertreated [12, 20].

Once PAD is diagnosed, all risk factors must be rigorously managed, which includes smoking cessation, administering aspirin, statins, and cilostazol, and undertaking active exercise $[19,22]$. Timely diagnosis of PAD and its early treatment can improve patients' quality of life and avoid or, at least, delay undesirable events, including amputation or death $[12,13]$.

\section{Study limitations}

This was a single-center study that involved a relatively small number of patients. Additionally, for the purposes of vascular access, ABI was calculated based on the systolic blood pressure that was measured on one side only, which may have affected the findings as a consequence of subclavian steal syndrome and underestimated the values in some patients. We evaluated left ventricular hypertrophy based on electrocardiography, and we did not perform echocardiography. Although we determined the total cholesterol and triglyceride values, there were no data that described the high- and low-density lipoprotein cholesterol levels. We performed a cross-sectional study of prevalent patients on HD; however, a long-term study that monitors the relationships between the risk factors and the development of PAD in incident patient's, would generate more robust data.

\section{Conclusion}

The prevalence of PAD was high in our group of patients on HD. Independent predictors of PAD were the presence of symptoms based on the Fontaine classification, a higher CRP level, and vascular access provided by a Hickman catheter for HD. An ABI $<0.9$ combined with the clinical findings and claudication more accurately signifies clinically significant PAD. An ABI $<0.9$ alone, without clinical findings, is not sufficient for the detection of clinically significant PAD in patients on HD. Adequate and timely diagnosis and treatment of PAD could improve the quality of life and postpone complications in these patients.

\section{Abbreviations \\ ABI: Ankle-brachial index; AVF: Arteriovenous fistula; AVG: Arteriovenous graft; BMI: Body mass index; CABG: Coronary artery bypass graft; CAD: Coronary artery disease; CRP: C-reactive protein; CVC: Central venous catheter; DOPPS: Dialysis Outcomes and Practice Patterns Study; DPA: Dorsalis pedis artery; HD: Hemodialysis; HEMO: Hemodialysis Study; LVH: Left ventricular hypertrophy; NHANES: National Health and Nutrition Examination Survey; PAD: Peripheral arterial disease; PCl: Percutaneous coronary intervention; TPA: Tibialis posterior artery}

Acknowledgements

We would like to thank Editage (http://www.editage.com) for English language editing.

\section{Authors' contributions}

RA conceived and designed the study, collecting data, performed the study, analyzed the data, and wrote the manuscript. ND conceived and designed the study, analyzed the data, prepare figures and tables and critical revision of the article. GT did statistical analysis, conceived and designed the study, analyzed the data, prepare figures and tables, reviewed drafts of the paper, BR did statistical analysis, prepare figures and tables. AJ data analysis and data interpretation, reviewed drafts of the paper. PĐ analyzed the data, reviewed drafts of the paper. $\mathrm{NI}$ conceived and designed the study, analyzed the data, critical revision and final approval of the article. All authors read and approved the final manuscript.

\section{Funding}

This research received no specific grant from any funding agency in the public, commercial, or not-for-profit sectors.

\section{Availability of data and materials}

The dataset used in a current study are available from the corresponding author on reasonable request. 


\section{Ethics approval and consent to participate}

Zvezdara University Medical Center's ethics committee approved this study (No IRB 00009457). All participants provided written informed consent before study initiation.

\section{Consent for publication}

Not applicable.

\section{Competing interests}

The authors declare that they have no competing interests.

\section{Author details}

${ }^{1}$ Department of Vascular Surgery Clinic of Surgery, Clinical Hospital Center Zvezdara, Dimitrija Tucovića 161, Belgrade 11000, Serbia. ${ }^{2}$ Clinic of Nephrology, Clinical Hospital Center Zvezdara, Belgrade, Serbia. ${ }^{3}$ Medical Faculty, University of Belgrade, Belgrade, Serbia. ${ }^{4}$ Department of Medical Statistics and Informatics Medical Faculty, University of Belgrade, Belgrade, Serbia. ${ }^{5}$ Primary Health Center, Palilula, Belgrade, Serbia. ${ }^{6}$ Vascular Surgery Clinic, Dedinje Cardiovascular Institute, Belgrade, Serbia.

Received: 21 February 2019 Accepted: 19 July 2019

Published online: 26 July 2019

\section{References}

1. Fowkes FGR, Rudan D, Rudan I, Aboyans V, Denenberg JO, McDermott MM, et al. Comparison of global estimates of prevalence and risk factors for peripheral artery disease in 2000 and 2010: a systematic review and analysis. Lancet. 2013;382(9901):1329-40.

2. Ohtake T, Oka M, Ikee R, Mochida Y, Ishioka K, Moriya H, et al. Impact of lower limbs' arterial calcification on the prevalence and severity of PAD in patients on hemodialysis. J Vasc Surg. 2011;53(3):676-83.

3. Viazzi F, Leoncini G, Ratto E, Storace G, Gonnella A, Garneri D, et al. Peripheral artery disease and blood pressure profile abnormalities in hemodialysis patients. J Nephrol. 2017;30(3):427-33.

4. Fowkes FGR, Aboyans V, Fowkes FJl, McDermott MM, Sampson UKA, Criqu MH. Peripheral artery disease: epidemiology and global perspectives. Nat Rev Cardiol. 2017:14(3):156-70.

5. Criqui MH, Aboyans V. Epidemiology of peripheral artery disease. Circ Res. 2015;116(9):1509-26.

6. Garimella PS, Hirsch AT. Peripheral artery disease and chronic kidney disease: clinical synergy to improve outcomes. Adv Chronic Dis. 2014; 21(6):460-71.

7. Herzog CA, Asinger RW, Berger AK, Charytan DM, Díez J, Hart RG, et al. Cardiovascular disease in chronic kidney disease. A clinical update from kidney disease: improving global outcomes (KDIGO). Kidney Int. 2011;80: 572-86.

8. Rajagopalan S, Dellegrottaglie S, Furniss AL, Gillespie BW, Satayathum S, Lameire $\mathrm{N}$, et al. Peripheral arterial disease in patients with end-stage renal disease: observations from the Dialysis outcomes and practice patterns study (DOPPS). Circulation. 2006;114(18):1914-22.

9. Cheung AK, Sarnak MJ, Yan G, Dwyer JT, Heyka RJ, Rocco MV, et al. Atherosclerotic cardiovascular disease risks in chronic hemodialysis patients. Kidney Int. 2000;58(1):353-62.

10. O'Hare A, Glidden D, Fox C, HSU C. High prevalence of peripheral arterial disease in persons with renal insufficiency: results from the National Health and nutrition examination survey 1999-2000. Circulation. 2004;109(3):320-3.

11. Ogata H, Kumata-Maeta C, Shishido K, Mizobuchi M, Yamamoto M, Koiwa F, et al. Detection of peripheral artery disease by duplex ultrasonography among hemodialysis patients. Clin J Am Soc Nephrol. 2010:5(12):2199-206.

12. Matsuzawa R, Aoyama N, Yoshida A. Clinical characteristics of patients on hemodialysis with peripheral arterial disease. Angiology. 2015;66(10):911-7.

13. Chen J, Mohler ER, Xie D, Shlipak M, Townsend RR, Appel L, et al. Traditional and non-traditional risk factors for incident peripheral arterial disease among patients with chronic kidney disease. Nephrol Dial Transplant. 2016;31(7):1145-51.

14. Combe C, Albert JM, Bragg-Gresham JL, Andreucci VE, Disney A, Fukuhara S, et al. The burden of amputation among hemodialysis patients in the Dialysis outcomes and practice patterns study (DOPPS). Am J Kidney Dis. 2009;54(4):680-92.

15. Fontaine R, Kim M, Kieny R. Surgical treatment of peripheral circulation disorders. Helv Chir Acta. 1954;21(5-6):499-533.
16. Adragao T, Pires A, Branco P, Castro R, Oliveira A, Nogueira C, et al. Anklebrachial index, vascular calcifications and mortality in dialysis patients. Nephrol Dial Transplant. 2012;27(1):318-25.

17. R Development Core Team. A language and environment for statistical computing. Vienna: R Foundation for Statistical Computing; 2017. Available from: https://www.r-project.org/

18. Aboyans V, Criqui MH, Abraham P, Allison MA, Creager MA, Diehm C, et al. Measurement and interpretation of the ankle-brachial index: a scientific statement from the American Heart Association. Circulation. 2012;126(24): 2890-909.

19. Yang Y, Ning Y, Shang W, Luo R, Li L, Guo S, et al. Association of peripheral arterial disease with all-cause and cardiovascular mortality in hemodialysis patients: a meta-analysis. BMC Nephrol. 2016;17(1):1-9.

20. McDermott MM. Lower extremity manifestations of peripheral artery disease: the pathophysiologic and functional implications of leg ischemia. Circ Res. 2015;116(9):1540-50.

21. Webb AT, Franks PJ, Reaveley DA, Greenhalgh RM, Brown EA. Prevalence of intermittent claudication and risk factors for its development in patients on renal replacement therapy. Eur J Vasc Surg. 1993 Sep;7(5):523-7.

22. Ishii H, Aoyama T, Takahashi H, Kumada Y, Kamoi D, Sakakibara T, et al. Treatment with cilostazol improves clinical outcome after endovascular therapy in hemodialysis patients with peripheral artery disease. J Cardiol. 2016;67(2):199-204.

23. Desormais I, Aboyans V, Bura A, Constans J, Cambou J-P, Messas E, et al. Anemia, an independent predictive factor for amputation and mortality in patients hospitalized for peripheral artery disease. Eur J Vasc Endovasc Surg. 2014 Aug;48(2):202-7.

24. Jaar BG, Plantinga LC, Astor BC, Fink NE, Longenecker C, Tracy RP, et al. Novel and traditional cardiovascular risk factors for peripheral arterial disease in incident-dialysis patients. Adv Chronic Kidney Dis. 2007 Jul; 14(3):304-13.

25. O'Hare AM, Hsu C, Bacchetti P, Johansen KL. Peripheral vascular disease risk factors among patients undergoing hemodialysis. J Am Soc Nephrol. 2002; 13(2):497-503.

26. Kaminski MR, Raspovic A, McMahon LP, Strippoli GFM, Palmer SC, Ruospo $M$, et al. Risk factors for foot ulceration and lower extremity amputation in adults with end-stage renal disease on dialysis: a systematic review and meta-analysis. Nephrol Dial Transplant. 2015;30(10):1747-66.

27. Ozaki Y, Imanishi T, Akasaka T. Inflammatory biomarkers in peripheral artery disease: diagnosis, prognosis, and therapeutic challenges. Curr Med Chem. 2015;22(23):2744-53

28. Stenvinkel P, Heimbürger $\mathrm{O}$, Lindholm B, Kaysen GA, Bergström J. Are there two types of malnutrition in chronic renal failure? Evidence for relationships between malnutrition, inflammation and atherosclerosis (MIA syndrome). Nephrol Dial Transplant. 2000;15(7):953-60.

29. Suominen V, Rantanen T, Venermo M, Saarinen J, Salenius J. Prevalence and risk factors of PAD among patients with elevated ABI. Eur J Vasc Endovasc Surg. 2008;35(6):709-14.

30. Pisoni RL, Young EW, Dykstra DM, Greenwood RN, Hecking E, Gillespie B, et al. Vascular access use in Europe and the United States: results from the DOPPS. Kidney Int. 2002;61 (1):305-16.

31. Chen S-C, Chang J-M, Hwang S-J, Tsai J-C, Wang C-S, Mai H-C, et al. Significant correlation between ankle-brachial index and vascular access failure in hemodialysis patients. Clin J Am Soc Nephrol. 2009;4(1):128-34.

32. Miyahara T, Suhara M, Nemoto $Y$, Shirasu T, Haga M, Mochizuki Y, et al. Long-Term Results of Treatment for Critical Limb Ischemia. Ann Vasc Dis. 2015;8:192-7.

33. National Kidney Foundation KDOQI Guidelines. KDOQI clinical practice guidelines for cardiovascular disease in Dialysis patients. Am J Kidney Dis. 2005;45(4):S1-153

\section{Publisher's Note}

Springer Nature remains neutral with regard to jurisdictional claims in published maps and institutional affiliations. 